\title{
Editorial
}

\section{Stomach and Duodenum: Special Issue-Part One}

\author{
Argha Chatterjee ${ }^{1}$ Rochita Venkataramanan ${ }^{2}$ \\ ${ }^{1}$ Department of Radiology and Imaging, Tata Medical Centre, \\ Kolkata, West Bengal, India \\ 2Department of Radiology, Apollo Hospitals, Chennai, Chennai, \\ Tamil Nadu, India
}

J Gastrointestinal Abdominal Radiol ISGAR 2021;4:83-84.

As we are slowly coming out of another wave of the pandemic and the chatter on COVID-19 gradually settles, it is perhaps a great time to brush up on some of the less illuminated areas of abdominal radiology that we face in our daily practice. Stomach and duodenum are among the most commonly overlooked regions in the era of cross-sectional imaging. Gastric pathologies, ranging from polyps to malignant neoplasms, are often missed in screening abdominal imaging. ${ }^{1}$ The fundus and antrum are particularly difficult areas to evaluate. ${ }^{2}$ Duodenum, despite its length and strategic position in the abdomen, is often ignored by radiologists. ${ }^{3}$ In yesteryears, barium study of the stomach and duodenum was the main diagnostic tool. However, with endoscopy taking the lead in evaluating the stomach and duodenum, radiologists find fewer and fewer referrals for imaging of these organs. Barium studies are now bordering on extinction. As a result, these regions do not always receive the attention they deserve in imaging literature. With this in mind, we are bringing out these two special issues where we attempt to focus on the stomach and duodenum.

In the first part, we review both conventional wisdom and new frontiers in gastric and duodenal imaging. The second part will largely focus on neoplastic conditions. In this part, Vora et al discussed the radiological anatomy of the stomach and duodenum with special emphasis on clinical aspects. ${ }^{4} \mathrm{~A}$ thorough discussion is included regarding the various imaging modalities employed in the pathologies of stomach and duodenum. Prithviraj et al have presented an extensive review of pediatric gastric and duodenal pathologies, starting from the very common hypertrophic pyloric stenosis to the very rare entities such as malignancies. ${ }^{5}$ Rastogi et al have discussed an important clinical condition, gastric outlet obstruction, in light of modern imaging philosophy. The emphasis given CT should prove very useful to our readers, as it reflects their current clinical practice. ${ }^{6}$ Finally, Girishekhar et al shed some light on the upcoming issue of bariatric surgery, which has now come to the realm of general radiology

DOI https://doi.org/ 10.1055/s-0041-1731957 ISSN 2581-9933.
Address for correspondence Argha Chatterjee, MD, PDCC, Department of Radiology and Imaging, Tata Medical Centre, 14 Main Arterial Road, Kolkata 700156, West Bengal, India (e-mail: arghachat84@gmail.com).

practice as well. ${ }^{7}$ The stress is on postoperative anatomy and its complications, and their exhaustive presentation of fluoroscopy is enlightening.

In harmony with the theme of the overlooked regions of the abdomen in this issue, Nadeem et al presented an exhaustive review of splenic pathologies. ${ }^{8}$ JGAR is continuing to observe an increase in the submission of original research. In this issue, Youseef et al presented a large series of perianal sepsis and discussed how transperineal, transvaginal and transabdominal ultrasound can be used to evaluate the nature and extent of intersphincteric plane infection. ${ }^{9}$ Shah et al presented two cases of mass-forming autoimmune pancreatitis and discussed the clinical dilemma in such cases. ${ }^{10}$ Their discussion on the role of biopsy and serum markers in such cases should prove very useful for the readers. Finally, the bizarre case of double gossypiboma, presented by Batra et al, reminds us of the importance of keeping our eyes open for uncommon possibilities over and above the usual suspects. ${ }^{11}$

We thank our readers, authors, reviewers, and editors for all of their valuable support. We look forward to more exciting research articles and excellent reviews.

\section{Conflict of Interest}

None declared.

\section{References}

1 Ivan CV, Mullineux JH, Shah V, Verma R, Rajesh A, Stephenson JA. Peripheral vision: abdominal pathology missed outside the centre of gaze. Br J Radiol 2018;91(1091) :20180142

2 Horton KM, Johnson PT, Fishman EK. MDCT of the abdomen: common misdiagnoses at a busy academic center. Am J Roentgenol 2010;194(3):660-667

3 Jayaraman MV, Mayo-Smith WW, Movson JS, Dupuy DE, Wallach MT. CT of the duodenum: an overlooked segment gets its due. Radiographics 2001;21(Spec No):S147-S160

4 Vora Z, Goyal A, Sharma R. Radiological anatomy of stomach and duodenum with clinical significance. J Gastrointestinal Abdominal Radiol ISGAR 2021;4(2):90-98 
5 Pritviraj SK, Kandasamy D, Jana M, Sharma R, Gupta AK. Pediatric stomach and duodenal imaging. J Gastrointestinal Abdominal Radiol ISGAR 2021;4(2):99-113

6 Rastogi A, Singh S, Yadav R. Gastric outlet obstruction in the current era-a pictorial review on computed tomography imaging. J Gastrointestinal Abdominal Radiol ISGAR 2021;4(2):144-153

7 Girishekar B, Rawat S, Ananthasivan R, Reddy P, Patil P, Kaushik K. Role of imaging in bariatric surgery: a review of the various surgical techniques and their complications. J Gastrointestinal Abdominal Radiol ISGAR 2021;4(2):114-131

8 Nadeem M, Tiwari HA, Jambhekar K, Shah H, Ram R. Revisiting the spleen-an imaging review of the common and uncommon splenic pathology. J Gastrointestinal Abdominal Radiol ISGAR 2021;4(2):132-143
9 Youssef AT. Significance and impact of the intersphincteric plane sepsis in the management of the perianal sepsis: ultrasound study. J Gastrointestinal Abdominal Radiol ISGAR 2021;4(2):85-89

10 Shah DS, Prajapati B, Sanghavi K, Kanhere S, Kothari J, Dubal J. Mass mimicking autoimmune pancreatitis- a report of two cases and review of literature. J Gastrointestinal Abdominal Radiol ISGAR 2021;4(2):154-160

11 Batra R, Gautam R, Manchanda A, Ghuliani D. A case of two abdominal gossypibomas in a patient: a rare case report. J Gastrointestinal Abdominal Radiol ISGAR 2021;4(2):161-165 